\title{
Hybridizing sequential and variational data assimilation for robust high-resolution hydrologic forecasting
}

Felipe Hernández, Xu Liang

Civil and Environmental Engineering Department, University of Pittsburgh, Pittsburgh, 15213, United States of America

5 Correspondence to: Xu Liang (xuliang@pitt.edu)

Supplemental information 
Table 1. Parameter assignments and error metrics for all the configurations of Experiment 1 (Blue River), Scenario 1 (November $1^{\text {th }}$, 1996). Configuration 0 corresponds to the default model. All other errors are computed using the OPTIMISTS ensemble mean.

\begin{tabular}{|c|c|c|c|c|c|c|c|c|c|c|c|}
\hline \multirow{2}{*}{ Config. } & \multirow{2}{*}{$\Delta t$} & \multirow{2}{*}{ Objs. } & \multirow{2}{*}{$n$} & \multirow{2}{*}{$p_{\text {samp }}$} & \multirow{2}{*}{$\boldsymbol{k}_{\mathrm{F} \text {-class }}$} & \multicolumn{3}{|c|}{ Assimilation period } & \multicolumn{3}{|c|}{ Forecast period } \\
\hline & & & & & & $\mathrm{NSE}_{12}$ & $\mathrm{NSE}_{\ell 1}$ & MARE & $\mathrm{NSE}_{12}$ & $\mathrm{NSE}_{\ell 1}$ & MARE \\
\hline 0 & & -- No da & a assir & nilation & & 0.801 & 0.613 & $26.2 \%$ & 0.553 & 0.404 & $16.5 \%$ \\
\hline 1 & $1 \mathrm{w}$ & 1 & 200 & 0.4 & no & 0.968 & 0.861 & $8.6 \%$ & -1.109 & -0.496 & $45.4 \%$ \\
\hline 2 & $1 \mathrm{w}$ & 1 & 200 & 0.4 & yes & 0.980 & 0.879 & $9.6 \%$ & -1.519 & -0.749 & $55.5 \%$ \\
\hline 3 & $1 \mathrm{w}$ & 1 & 200 & 1.0 & no & 0.899 & 0.746 & $15.3 \%$ & -1.455 & -0.519 & $43.9 \%$ \\
\hline 4 & $1 \mathrm{w}$ & 1 & 200 & 1.0 & yes & 0.960 & 0.828 & $11.4 \%$ & -0.465 & -0.348 & $42.7 \%$ \\
\hline 5 & $2 \mathrm{w}$ & 1 & 200 & 0.4 & no & 0.922 & 0.798 & $12.3 \%$ & -1.287 & -0.564 & $47.6 \%$ \\
\hline 6 & $2 \mathrm{w}$ & 1 & 200 & 0.4 & yes & 0.964 & 0.823 & $13.3 \%$ & -0.353 & -0.343 & $44.1 \%$ \\
\hline 7 & $2 w$ & 1 & 200 & 1.0 & no & 0.877 & 0.721 & $16.9 \%$ & -1.252 & -0.453 & $42.0 \%$ \\
\hline 8 & $2 w$ & 1 & 200 & 1.0 & yes & 0.929 & 0.770 & $15.9 \%$ & -0.502 & -0.271 & $38.5 \%$ \\
\hline 9 & $1 \mathrm{w}$ & 1 & 500 & 0.4 & no & 0.952 & 0.823 & $13.6 \%$ & -1.947 & -1.003 & $67.2 \%$ \\
\hline 10 & $1 \mathrm{w}$ & 1 & 500 & 0.4 & yes & 0.969 & 0.851 & $8.7 \%$ & -0.509 & -0.405 & $45.4 \%$ \\
\hline 11 & $1 \mathrm{w}$ & 1 & 500 & 1.0 & no & 0.896 & 0.749 & $14.7 \%$ & -1.552 & -0.591 & $46.9 \%$ \\
\hline 12 & $1 \mathrm{w}$ & 1 & 500 & 1.0 & yes & 0.970 & 0.846 & $10.9 \%$ & -1.349 & -0.538 & $45.3 \%$ \\
\hline 13 & $2 \mathrm{w}$ & 1 & 500 & 0.4 & no & 0.955 & 0.831 & $10.6 \%$ & -1.616 & -0.500 & $42.0 \%$ \\
\hline 14 & $2 \mathrm{w}$ & 1 & 500 & 0.4 & yes & 0.969 & 0.843 & $12.5 \%$ & -0.488 & -0.360 & $44.7 \%$ \\
\hline 15 & $2 w$ & 1 & 500 & 1.0 & no & 0.884 & 0.733 & $16.0 \%$ & -1.314 & -0.458 & $42.0 \%$ \\
\hline 16 & $2 \mathrm{w}$ & 1 & 500 & 1.0 & yes & 0.937 & 0.778 & $16.1 \%$ & -0.718 & -0.356 & $41.1 \%$ \\
\hline 17 & $1 \mathrm{w}$ & 2 & 200 & 0.4 & no & 0.927 & 0.784 & $12.7 \%$ & -1.697 & -0.664 & $49.3 \%$ \\
\hline 18 & $1 \mathrm{w}$ & 2 & 200 & 0.4 & yes & 0.946 & 0.813 & $10.2 \%$ & -2.005 & -0.786 & $54.0 \%$ \\
\hline 19 & $1 \mathrm{w}$ & 2 & 200 & 1.0 & no & 0.873 & 0.720 & $17.5 \%$ & -1.256 & -0.520 & $45.3 \%$ \\
\hline 20 & $1 \mathrm{w}$ & 2 & 200 & 1.0 & yes & 0.930 & 0.778 & $13.3 \%$ & -1.141 & -0.419 & $40.9 \%$ \\
\hline 21 & $2 \mathrm{w}$ & 2 & 200 & 0.4 & no & 0.914 & 0.763 & $13.8 \%$ & -1.179 & -0.403 & $40.2 \%$ \\
\hline 22 & $2 w$ & 2 & 200 & 0.4 & yes & 0.889 & 0.731 & $15.7 \%$ & -1.138 & -0.439 & $42.2 \%$ \\
\hline 23 & $2 \mathrm{w}$ & 2 & 200 & 1.0 & no & 0.846 & 0.692 & $18.7 \%$ & -1.189 & -0.446 & $42.2 \%$ \\
\hline 24 & $2 \mathrm{w}$ & 2 & 200 & 1.0 & yes & 0.875 & 0.718 & $16.7 \%$ & -1.244 & -0.473 & $43.3 \%$ \\
\hline 25 & $1 \mathrm{w}$ & 2 & 500 & 0.4 & no & 0.951 & 0.816 & $12.6 \%$ & -1.482 & -0.850 & $61.4 \%$ \\
\hline 26 & $1 \mathrm{w}$ & 2 & 500 & 0.4 & yes & 0.960 & 0.825 & $11.3 \%$ & 0.015 & -0.080 & $33.9 \%$ \\
\hline 27 & $1 \mathrm{w}$ & 2 & 500 & 1.0 & no & 0.866 & 0.714 & $17.3 \%$ & -1.434 & -0.554 & $45.9 \%$ \\
\hline 28 & $1 \mathrm{w}$ & 2 & 500 & 1.0 & yes & 0.913 & 0.765 & $14.7 \%$ & -1.603 & -0.554 & $44.8 \%$ \\
\hline 29 & $2 \mathrm{w}$ & 2 & 500 & 0.4 & no & 0.890 & 0.733 & $16.8 \%$ & -1.258 & -0.492 & $44.0 \%$ \\
\hline 30 & $2 \mathrm{w}$ & 2 & 500 & 0.4 & yes & 0.905 & 0.747 & $15.5 \%$ & -1.130 & -0.466 & $43.8 \%$ \\
\hline 31 & $2 w$ & 2 & 500 & 1.0 & no & 0.860 & 0.703 & $17.7 \%$ & -1.117 & -0.424 & $41.6 \%$ \\
\hline 32 & $2 w$ & 2 & 500 & 1.0 & yes & 0.881 & 0.723 & $16.4 \%$ & -1.150 & -0.449 & $42.7 \%$ \\
\hline
\end{tabular}


Table 2. Parameter assignments and error metrics for all the configurations of Experiment 1 (Blue River), Scenario 2 (April $8^{\text {th }}$, 1997). Configuration 0 corresponds to the default model. All other errors are computed using the OPTIMISTS ensemble mean.

\begin{tabular}{|c|c|c|c|c|c|c|c|c|c|c|c|}
\hline \multirow{2}{*}{ Config. } & \multirow{2}{*}{$\Delta t$} & \multirow{2}{*}{ Objs. } & \multirow{2}{*}{$n$} & \multirow{2}{*}{$p_{\text {samp }}$} & \multirow{2}{*}{$k_{\text {F-class }}$} & \multicolumn{3}{|c|}{ Assimilation period } & \multicolumn{3}{|c|}{ Forecast period } \\
\hline & & & & & & $\mathrm{NSE}_{12}$ & 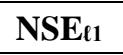 & MARE & $\mathrm{NSE}_{12}$ & $\mathrm{NSE}_{t 1}$ & MARE \\
\hline 0 & & -- No da & a assir & nilation & & -0.249 & -0.641 & $122.5 \%$ & 0.498 & 0.108 & $106.8 \%$ \\
\hline 1 & $1 \mathrm{w}$ & 1 & 200 & 0.4 & no & 0.402 & -0.16 & $80.7 \%$ & 0.479 & 0.129 & $99.7 \%$ \\
\hline 2 & $1 \mathrm{w}$ & 1 & 200 & 0.4 & yes & 0.505 & -0.023 & $70.9 \%$ & -0.083 & -0.061 & $110.7 \%$ \\
\hline 3 & $1 \mathrm{w}$ & 1 & 200 & 1 & no & 0.285 & -0.285 & $91.6 \%$ & 0.435 & -0.003 & $111.5 \%$ \\
\hline 4 & $1 \mathrm{w}$ & 1 & 200 & 1 & yes & 0.283 & -0.161 & $81.0 \%$ & 0.555 & 0.179 & $79.9 \%$ \\
\hline 5 & $2 w$ & 1 & 200 & 0.4 & no & 0.387 & -0.177 & $82.5 \%$ & 0.580 & 0.144 & $92.3 \%$ \\
\hline 6 & $2 w$ & 1 & 200 & 0.4 & yes & 0.307 & -0.126 & $78.0 \%$ & 0.629 & 0.207 & $82.2 \%$ \\
\hline 7 & $2 \mathrm{w}$ & 1 & 200 & 1 & no & 0.324 & -0.245 & $88.1 \%$ & 0.512 & 0.067 & $103.1 \%$ \\
\hline 8 & $2 w$ & 1 & 200 & 1 & yes & 0.287 & -0.142 & $79.5 \%$ & 0.552 & 0.157 & $83.7 \%$ \\
\hline 9 & $1 \mathrm{w}$ & 1 & 500 & 0.4 & no & 0.473 & -0.078 & $75.2 \%$ & 0.592 & 0.215 & $90.0 \%$ \\
\hline 10 & $1 \mathrm{w}$ & 1 & 500 & 0.4 & yes & 0.636 & 0.373 & $37.4 \%$ & 0.371 & 0.119 & $81.4 \%$ \\
\hline 11 & $1 \mathrm{w}$ & 1 & 500 & 1 & no & 0.323 & -0.258 & $90.1 \%$ & 0.488 & 0.052 & $107.2 \%$ \\
\hline 12 & $1 \mathrm{w}$ & 1 & 500 & 1 & yes & 0.279 & -0.123 & $78.2 \%$ & 0.508 & 0.187 & $74.0 \%$ \\
\hline 13 & $2 \mathrm{w}$ & 1 & 500 & 0.4 & no & 0.453 & -0.087 & $79.9 \%$ & 0.246 & -0.043 & $113.9 \%$ \\
\hline 14 & $2 w$ & 1 & 500 & 0.4 & yes & 0.503 & 0.026 & $66.9 \%$ & 0.600 & 0.213 & $83.4 \%$ \\
\hline 15 & $2 \mathrm{w}$ & 1 & 500 & 1 & no & 0.343 & -0.231 & $87.6 \%$ & 0.497 & 0.054 & $102.8 \%$ \\
\hline 16 & $2 w$ & 1 & 500 & 1 & yes & 0.284 & -0.088 & $74.4 \%$ & 0.573 & 0.224 & $72.9 \%$ \\
\hline 17 & $1 \mathrm{w}$ & 2 & 200 & 0.4 & no & -0.381 & -0.69 & $124.0 \%$ & 0.427 & -0.007 & $113.3 \%$ \\
\hline 18 & $1 \mathrm{w}$ & 2 & 200 & 0.4 & yes & 0.39 & -0.148 & $79.9 \%$ & 0.579 & 0.242 & $70.1 \%$ \\
\hline 19 & $1 \mathrm{w}$ & 2 & 200 & 1 & no & -0.435 & -0.703 & $124.8 \%$ & 0.413 & -0.022 & $114.7 \%$ \\
\hline 20 & $1 \mathrm{w}$ & 2 & 200 & 1 & yes & 0.566 & 0.125 & $56.8 \%$ & 0.542 & 0.404 & $41.1 \%$ \\
\hline 21 & $2 \mathrm{w}$ & 2 & 200 & 0.4 & no & -0.76 & -0.823 & $133.7 \%$ & 0.425 & 0.002 & $114.9 \%$ \\
\hline 22 & $2 \mathrm{w}$ & 2 & 200 & 0.4 & yes & 0.311 & -0.162 & $80.6 \%$ & 0.592 & 0.170 & $85.4 \%$ \\
\hline 23 & $2 \mathrm{w}$ & 2 & 200 & 1 & no & -0.677 & -0.781 & $131.1 \%$ & 0.428 & 0.000 & $114.0 \%$ \\
\hline 24 & $2 \mathrm{w}$ & 2 & 200 & 1 & yes & 0.284 & -0.263 & $89.6 \%$ & 0.517 & 0.073 & $100.8 \%$ \\
\hline 25 & $1 \mathrm{w}$ & 2 & 500 & 0.4 & no & -0.116 & -0.528 & $110.9 \%$ & 0.504 & 0.072 & $99.5 \%$ \\
\hline 26 & $1 \mathrm{w}$ & 2 & 500 & 0.4 & yes & 0.502 & 0.089 & $59.4 \%$ & 0.463 & 0.365 & $43.8 \%$ \\
\hline 27 & $1 \mathrm{w}$ & 2 & 500 & 1 & no & -0.324 & -0.652 & $121.1 \%$ & 0.449 & 0.017 & $112.2 \%$ \\
\hline 28 & $1 \mathrm{w}$ & 2 & 500 & 1 & yes & 0.467 & 0.067 & $60.4 \%$ & 0.458 & 0.383 & $39.4 \%$ \\
\hline 29 & $2 w$ & 2 & 500 & 0.4 & no & -0.174 & -0.572 & $113.9 \%$ & 0.460 & 0.038 & $110.5 \%$ \\
\hline 30 & $2 w$ & 2 & 500 & 0.4 & yes & 0.326 & -0.203 & $84.6 \%$ & 0.571 & 0.135 & $94.0 \%$ \\
\hline 31 & $2 w$ & 2 & 500 & 1 & no & -0.457 & -0.701 & $124.3 \%$ & 0.445 & 0.014 & $112.1 \%$ \\
\hline 32 & $2 w$ & 2 & 500 & 1 & yes & 0.304 & -0.209 & $85.0 \%$ & 0.538 & 0.101 & $95.1 \%$ \\
\hline
\end{tabular}


Table 3. Parameter assignments and error metrics for all the configurations of Experiment 1 (Blue River), Scenario 2 (February $\left.24^{\text {th }}, \mathbf{1 9 9 8}\right)$. Configuration 0 corresponds to the default model. All other errors are computed using the OPTIMISTS ensemble mean.

\begin{tabular}{|c|c|c|c|c|c|c|c|c|c|c|c|}
\hline \multirow{2}{*}{ Config. } & \multirow{2}{*}{$\Delta t$} & \multirow{2}{*}{ Objs. } & \multirow{2}{*}{$n$} & \multirow{2}{*}{$p_{\text {samp }}$} & \multirow{2}{*}{$k_{\mathrm{F} \text {-class }}$} & \multicolumn{3}{|c|}{ Assimilation period } & \multicolumn{3}{|c|}{ Forecast period } \\
\hline & & & & & & $\mathrm{NSE}_{12}$ & $\mathrm{NSE}_{\ell 1}$ & MARE & $\mathrm{NSE}_{12}$ & $\mathrm{NSE}_{\ell 1}$ & MARE \\
\hline 0 & & - No da & a assir & iilation & - & 0.608 & 0.174 & $141.7 \%$ & 0.758 & 0.480 & $101.7 \%$ \\
\hline 1 & $1 \mathrm{w}$ & 1 & 200 & 0.4 & no & 0.689 & 0.387 & $92.2 \%$ & 0.864 & 0.609 & $81.6 \%$ \\
\hline 2 & $1 \mathrm{w}$ & 1 & 200 & 0.4 & yes & 0.608 & 0.361 & $96.0 \%$ & 0.885 & 0.662 & $73.4 \%$ \\
\hline 3 & $1 \mathrm{w}$ & 1 & 200 & 1 & no & 0.66 & 0.365 & $98.0 \%$ & 0.871 & 0.594 & $85.2 \%$ \\
\hline 4 & $1 \mathrm{w}$ & 1 & 200 & 1 & yes & 0.586 & 0.376 & $89.5 \%$ & 0.824 & 0.619 & $71.3 \%$ \\
\hline 5 & $2 \mathrm{w}$ & 1 & 200 & 0.4 & no & 0.648 & 0.346 & $100.8 \%$ & 0.866 & 0.603 & $72.8 \%$ \\
\hline 6 & $2 w$ & 1 & 200 & 0.4 & yes & 0.57 & 0.329 & $99.4 \%$ & 0.884 & 0.635 & $72.1 \%$ \\
\hline 7 & $2 w$ & 1 & 200 & 1 & no & 0.606 & 0.329 & $101.8 \%$ & 0.886 & 0.630 & $74.1 \%$ \\
\hline 8 & $2 w$ & 1 & 200 & 1 & yes & 0.539 & 0.299 & $104.8 \%$ & 0.887 & 0.637 & $73.5 \%$ \\
\hline 9 & $1 \mathrm{w}$ & 1 & 500 & 0.4 & no & 0.74 & 0.437 & $85.5 \%$ & 0.858 & 0.594 & $75.4 \%$ \\
\hline 10 & $1 \mathrm{w}$ & 1 & 500 & 0.4 & yes & 0.691 & 0.489 & $62.9 \%$ & 0.865 & 0.645 & $70.9 \%$ \\
\hline 11 & $1 \mathrm{w}$ & 1 & 500 & 1 & no & 0.656 & 0.375 & $98.3 \%$ & 0.874 & 0.603 & $85.6 \%$ \\
\hline 12 & $1 \mathrm{w}$ & 1 & 500 & 1 & yes & 0.611 & 0.373 & $92.1 \%$ & 0.861 & 0.645 & $72.0 \%$ \\
\hline 13 & $2 w$ & 1 & 500 & 0.4 & no & 0.623 & 0.379 & $91.9 \%$ & 0.894 & 0.636 & $72.5 \%$ \\
\hline 14 & $2 \mathrm{w}$ & 1 & 500 & 0.4 & yes & 0.682 & 0.381 & $94.2 \%$ & 0.875 & 0.630 & $66.2 \%$ \\
\hline 15 & $2 \mathrm{w}$ & 1 & 500 & 1 & no & 0.613 & 0.339 & $100.3 \%$ & 0.892 & 0.632 & $72.5 \%$ \\
\hline 16 & $2 \mathrm{w}$ & 1 & 500 & 1 & yes & 0.532 & 0.301 & $104.1 \%$ & 0.889 & 0.643 & $72.9 \%$ \\
\hline 17 & $1 \mathrm{w}$ & 2 & 200 & 0.4 & no & 0.631 & 0.233 & $122.2 \%$ & 0.849 & 0.560 & $90.0 \%$ \\
\hline 18 & $1 \mathrm{w}$ & 2 & 200 & 0.4 & yes & 0.69 & 0.339 & $105.8 \%$ & 0.813 & 0.525 & $94.0 \%$ \\
\hline 19 & $1 \mathrm{w}$ & 2 & 200 & 1 & no & 0.667 & 0.284 & $114.6 \%$ & 0.853 & 0.566 & $91.2 \%$ \\
\hline 20 & $1 \mathrm{w}$ & 2 & 200 & 1 & yes & 0.67 & 0.351 & $98.9 \%$ & 0.874 & 0.603 & $81.9 \%$ \\
\hline 21 & $2 w$ & 2 & 200 & 0.4 & no & 0.588 & 0.1 & $144.7 \%$ & 0.823 & 0.555 & $80.3 \%$ \\
\hline 22 & $2 w$ & 2 & 200 & 0.4 & yes & 0.586 & 0.297 & $106.7 \%$ & 0.886 & 0.632 & $73.4 \%$ \\
\hline 23 & $2 w$ & 2 & 200 & 1 & no & 0.602 & 0.106 & $145.1 \%$ & 0.814 & 0.545 & $82.0 \%$ \\
\hline 24 & $2 w$ & 2 & 200 & 1 & yes & 0.571 & 0.289 & $108.7 \%$ & 0.880 & 0.619 & $75.2 \%$ \\
\hline 25 & $1 \mathrm{w}$ & 2 & 500 & 0.4 & no & 0.623 & 0.258 & $120.8 \%$ & 0.852 & 0.571 & $87.0 \%$ \\
\hline 26 & $1 \mathrm{w}$ & 2 & 500 & 0.4 & yes & 0.705 & 0.369 & $98.5 \%$ & 0.852 & 0.560 & $93.3 \%$ \\
\hline 27 & $1 \mathrm{w}$ & 2 & 500 & 1 & no & 0.637 & 0.269 & $117.6 \%$ & 0.851 & 0.565 & $88.6 \%$ \\
\hline 28 & $1 \mathrm{w}$ & 2 & 500 & 1 & yes & 0.675 & 0.347 & $101.3 \%$ & 0.858 & 0.576 & $88.5 \%$ \\
\hline 29 & $2 \mathrm{w}$ & 2 & 500 & 0.4 & no & 0.634 & 0.198 & $127.5 \%$ & 0.880 & 0.613 & $74.8 \%$ \\
\hline 30 & $2 w$ & 2 & 500 & 0.4 & yes & 0.56 & 0.308 & $103.3 \%$ & 0.884 & 0.629 & $75.5 \%$ \\
\hline 31 & $2 \mathrm{w}$ & 2 & 500 & 1 & no & 0.61 & 0.133 & $140.7 \%$ & 0.822 & 0.550 & $82.1 \%$ \\
\hline 32 & $2 w$ & 2 & 500 & 1 & yes & 0.54 & 0.298 & $105.1 \%$ & 0.886 & 0.634 & $73.7 \%$ \\
\hline
\end{tabular}


Table 4. Parameter assignments and error metrics for all the configurations of Experiment 2 (Indiantown Run), Scenario 1 (July $\left.2^{\text {th }}, 2009\right)$. Configuration 0 corresponds to the default model. All other errors are computed using the OPTIMISTS ensemble mean.

\begin{tabular}{|c|c|c|c|c|c|c|c|c|c|c|c|}
\hline \multirow{2}{*}{ Config. } & \multirow{2}{*}{$\Delta t$} & \multirow{2}{*}{ Objs. } & \multirow{2}{*}{$n$} & \multirow{2}{*}{$p_{\text {samp }}$} & \multirow{2}{*}{$w_{\text {root }}$} & \multicolumn{3}{|c|}{ Assimilation period } & \multicolumn{3}{|c|}{ Forecast period } \\
\hline & & & & & & $\mathrm{NSE}_{12}$ & $\mathrm{NSE}_{t 1}$ & MARE & $\mathrm{NSE}_{12}$ & $\mathrm{NSE}_{\ell 1}$ & MARE \\
\hline 0 & & - No dat & $\operatorname{assin}$ & ilation & & 0.478 & -0.015 & $24.9 \%$ & 0.022 & -0.003 & $54.3 \%$ \\
\hline 1 & $1 \mathrm{~h}$ & 2 & 100 & 0.25 & $60 \%$ & $-9 \mathrm{E}+04$ & $-1 E+02$ & $2.8 \mathrm{E}+01$ & 0.067 & -0.759 & $197.1 \%$ \\
\hline 2 & $1 \mathrm{~h}$ & 2 & 100 & 0.25 & $95 \%$ & $-2 \mathrm{E}+03$ & $-3 E+01$ & $7.4 \mathrm{E}+00$ & 0.365 & 0.193 & $73.5 \%$ \\
\hline 3 & $1 \mathrm{~h}$ & 2 & 100 & 1.00 & $60 \%$ & $-1 \mathrm{E}+01$ & -3.324 & $98.3 \%$ & 0.279 & -0.360 & $143.9 \%$ \\
\hline 4 & $1 \mathrm{~h}$ & 2 & 100 & 1.00 & $95 \%$ & -1.455 & -0.862 & $44.9 \%$ & 0.346 & -0.099 & $107.0 \%$ \\
\hline 5 & $1 \mathrm{~h}$ & 2 & 200 & 0.25 & $60 \%$ & $-2 \mathrm{E}+05$ & $-1 \mathrm{E}+02$ & $3.4 \mathrm{E}+01$ & 0.075 & -0.769 & $196.1 \%$ \\
\hline 6 & $1 \mathrm{~h}$ & 2 & 200 & 0.25 & $95 \%$ & $-3 E+03$ & $-3 E+01$ & $6.8 \mathrm{E}+00$ & 0.357 & 0.275 & $62.2 \%$ \\
\hline 7 & $1 \mathrm{~h}$ & 2 & 200 & 1.00 & $60 \%$ & $-1 \mathrm{E}+01$ & -3.900 & $111.6 \%$ & 0.284 & -0.330 & $143.5 \%$ \\
\hline 8 & $1 \mathrm{~h}$ & 2 & 200 & 1.00 & $95 \%$ & -2.320 & -1.192 & $54.1 \%$ & 0.341 & -0.182 & $120.1 \%$ \\
\hline 9 & $2 w$ & 2 & 100 & 0.25 & $60 \%$ & 0.471 & 0.208 & $18.1 \%$ & 0.317 & 0.381 & $43.2 \%$ \\
\hline 10 & $2 w$ & 2 & 100 & 0.25 & $95 \%$ & 0.099 & -0.001 & $23.2 \%$ & 0.320 & 0.375 & $44.3 \%$ \\
\hline 11 & $2 w$ & 2 & 100 & 1.00 & $60 \%$ & 0.571 & 0.371 & $13.3 \%$ & 0.292 & 0.393 & $38.3 \%$ \\
\hline 12 & $2 \mathrm{w}$ & 2 & 100 & 1.00 & $95 \%$ & 0.572 & 0.353 & $14.1 \%$ & 0.323 & 0.373 & $45.7 \%$ \\
\hline 13 & $2 \mathrm{w}$ & 2 & 200 & 0.25 & $60 \%$ & 0.531 & 0.295 & $15.5 \%$ & 0.327 & 0.367 & $47.0 \%$ \\
\hline 14 & $2 w$ & 2 & 200 & 0.25 & $95 \%$ & 0.520 & 0.305 & $15.4 \%$ & 0.312 & 0.380 & $42.6 \%$ \\
\hline 15 & $2 w$ & 2 & 200 & 1.00 & $60 \%$ & 0.360 & 0.211 & $17.5 \%$ & 0.322 & 0.338 & $50.1 \%$ \\
\hline 16 & $2 w$ & 2 & 200 & 1.00 & $95 \%$ & 0.427 & 0.175 & $18.7 \%$ & 0.332 & 0.357 & $49.0 \%$ \\
\hline 17 & $1 \mathrm{~h}$ & 1 & 100 & 0.25 & $60 \%$ & $-5 E+02$ & $-3 E+01$ & $7.0 \mathrm{E}+00$ & -0.429 & -1.338 & $2.8 \mathrm{E}+00$ \\
\hline 18 & $1 \mathrm{~h}$ & 1 & 100 & 0.25 & $95 \%$ & $-5 E+01$ & -7.655 & $196.9 \%$ & 0.094 & -0.736 & $193.1 \%$ \\
\hline 19 & $1 \mathrm{~h}$ & 1 & 100 & 1.00 & $60 \%$ & $-1 E+01$ & -3.201 & $96.0 \%$ & 0.314 & -0.269 & $132.4 \%$ \\
\hline 20 & $1 \mathrm{~h}$ & 1 & 100 & 1.00 & $95 \%$ & -9.818 & -3.016 & $90.8 \%$ & 0.290 & -0.354 & $142.2 \%$ \\
\hline 21 & $1 \mathrm{~h}$ & 1 & 200 & 0.25 & $60 \%$ & $-2 \mathrm{E}+05$ & $-6 E+02$ & $1.4 \mathrm{E}+02$ & $-5 E+01$ & -7.699 & $1.4 \mathrm{E}+01$ \\
\hline 22 & $1 \mathrm{~h}$ & 1 & 200 & 0.25 & $95 \%$ & $-1 \mathrm{E}+05$ & $-4 \mathrm{E}+02$ & $9.7 \mathrm{E}+01$ & $-5 \mathrm{E}+01$ & -7.337 & $1.4 \mathrm{E}+01$ \\
\hline 23 & $1 \mathrm{~h}$ & 1 & 200 & 1.00 & $60 \%$ & $-1 \mathrm{E}+01$ & -3.965 & $113.2 \%$ & 0.254 & -0.447 & $153.8 \%$ \\
\hline 24 & $1 \mathrm{~h}$ & 1 & 200 & 1.00 & $95 \%$ & $-1 \mathrm{E}+01$ & -3.601 & $103.3 \%$ & 0.283 & -0.374 & $144.9 \%$ \\
\hline 25 & $2 w$ & 1 & 100 & 0.25 & $60 \%$ & 0.453 & 0.071 & $21.8 \%$ & 0.320 & 0.373 & $44.2 \%$ \\
\hline 26 & $2 w$ & 1 & 100 & 0.25 & $95 \%$ & 0.279 & -0.053 & $24.6 \%$ & 0.329 & 0.368 & $46.3 \%$ \\
\hline 27 & $2 w$ & 1 & 100 & 1.00 & $60 \%$ & 0.500 & 0.177 & $18.9 \%$ & 0.331 & 0.369 & $46.3 \%$ \\
\hline 28 & $2 \mathrm{w}$ & 1 & 100 & 1.00 & $95 \%$ & 0.358 & 0.041 & $22.2 \%$ & 0.330 & 0.364 & $47.4 \%$ \\
\hline 29 & $2 w$ & 1 & 200 & 0.25 & $60 \%$ & 0.494 & 0.177 & $18.8 \%$ & 0.324 & 0.366 & $46.3 \%$ \\
\hline 30 & $2 \mathrm{w}$ & 1 & 200 & 0.25 & $95 \%$ & 0.362 & 0.036 & $22.3 \%$ & 0.329 & 0.362 & $47.4 \%$ \\
\hline 31 & $2 \mathrm{w}$ & 1 & 200 & 1.00 & $60 \%$ & 0.452 & 0.158 & $19.2 \%$ & 0.329 & 0.362 & $46.7 \%$ \\
\hline 32 & $2 \mathrm{w}$ & 1 & 200 & 1.00 & $95 \%$ & 0.480 & 0.155 & $19.4 \%$ & 0.333 & 0.362 & $47.2 \%$ \\
\hline
\end{tabular}


Table 5. Parameter assignments and error metrics for all the configurations of Experiment 2 (Indiantown Run), Scenario 2 (August $\left.2^{\text {th }}, 2009\right)$. Configuration 0 corresponds to the default model. All other errors are computed using the OPTIMISTS ensemble mean.

\begin{tabular}{|c|c|c|c|c|c|c|c|c|c|c|c|}
\hline \multirow{2}{*}{ Config. } & \multirow{2}{*}{$\Delta t$} & \multirow{2}{*}{ Objs. } & \multirow{2}{*}{$n$} & \multirow{2}{*}{$p_{\text {samp }}$} & \multirow{2}{*}{$w_{\text {root }}$} & \multicolumn{3}{|c|}{ Assimilation period } & \multicolumn{3}{|c|}{ Forecast period } \\
\hline & & & & & & $\mathrm{NSE}_{12}$ & $\mathrm{NSE}_{t 1}$ & MARE & $\mathrm{NSE}_{12}$ & $\mathrm{NSE}_{\ell 1}$ & MARE \\
\hline 0 & & - No dat & $\operatorname{assim}$ & ilation & & -0.926 & -0.963 & $68.0 \%$ & -0.428 & -0.733 & $58.3 \%$ \\
\hline 1 & $1 \mathrm{~h}$ & 2 & 100 & 0.25 & $60 \%$ & $-8 \mathrm{E}+02$ & $-2 E+01$ & $8.5 \mathrm{E}+00$ & -5.171 & -2.290 & $104.3 \%$ \\
\hline 2 & $1 \mathrm{~h}$ & 2 & 100 & 0.25 & $95 \%$ & $-2 \mathrm{E}+02$ & -7.769 & $3.9 \mathrm{E}+00$ & -0.581 & -0.517 & $46.2 \%$ \\
\hline 3 & $1 \mathrm{~h}$ & 2 & 100 & 1.00 & $60 \%$ & 0.672 & 0.570 & $16.1 \%$ & -1.459 & -1.065 & $66.7 \%$ \\
\hline 4 & $1 \mathrm{~h}$ & 2 & 100 & 1.00 & $95 \%$ & 0.637 & 0.643 & $11.4 \%$ & -0.971 & -0.799 & $57.0 \%$ \\
\hline 5 & $1 \mathrm{~h}$ & 2 & 200 & 0.25 & $60 \%$ & $-5 \mathrm{E}+03$ & $-3 \mathrm{E}+01$ & $1.0 \mathrm{E}+01$ & $-1 \mathrm{E}+01$ & -3.291 & $134.0 \%$ \\
\hline 6 & $1 \mathrm{~h}$ & 2 & 200 & 0.25 & $95 \%$ & $-3 \mathrm{E}+02$ & -9.816 & $5.0 \mathrm{E}+00$ & -1.156 & -0.797 & $54.9 \%$ \\
\hline 7 & $1 \mathrm{~h}$ & 2 & 200 & 1.00 & $60 \%$ & 0.708 & 0.518 & $19.2 \%$ & -1.493 & -1.082 & $67.2 \%$ \\
\hline 8 & $1 \mathrm{~h}$ & 2 & 200 & 1.00 & $95 \%$ & 0.657 & 0.625 & $12.9 \%$ & -1.259 & -0.966 & $63.0 \%$ \\
\hline 9 & $2 w$ & 2 & 100 & 0.25 & $60 \%$ & -0.607 & -0.154 & $35.9 \%$ & 0.322 & 0.118 & $25.8 \%$ \\
\hline 10 & $2 \mathrm{w}$ & 2 & 100 & 0.25 & $95 \%$ & 0.041 & 0.013 & $29.1 \%$ & 0.248 & 0.070 & $27.1 \%$ \\
\hline 11 & $2 w$ & 2 & 100 & 1.00 & $60 \%$ & 0.122 & 0.096 & $25.6 \%$ & 0.048 & -0.067 & $31.1 \%$ \\
\hline 12 & $2 \mathrm{w}$ & 2 & 100 & 1.00 & $95 \%$ & -0.113 & -0.164 & $35.1 \%$ & 0.451 & 0.212 & $23.2 \%$ \\
\hline 13 & $2 w$ & 2 & 200 & 0.25 & $60 \%$ & 0.186 & 0.161 & $23.5 \%$ & 0.051 & -0.089 & $32.1 \%$ \\
\hline 14 & $2 w$ & 2 & 200 & 0.25 & $95 \%$ & 0.118 & 0.088 & $26.0 \%$ & 0.132 & -0.010 & $29.4 \%$ \\
\hline 15 & $2 w$ & 2 & 200 & 1.00 & $60 \%$ & -0.019 & -0.052 & $30.9 \%$ & 0.255 & 0.077 & $26.9 \%$ \\
\hline 16 & $2 w$ & 2 & 200 & 1.00 & $95 \%$ & 0.022 & -0.030 & $30.3 \%$ & 0.279 & 0.085 & $26.8 \%$ \\
\hline 17 & $1 \mathrm{~h}$ & 1 & 100 & 0.25 & $60 \%$ & -3.015 & -2.226 & $138.7 \%$ & $-2 \mathrm{E}+01$ & -4.216 & $164.2 \%$ \\
\hline 18 & $1 \mathrm{~h}$ & 1 & 100 & 0.25 & $95 \%$ & -0.028 & -0.550 & $61.0 \%$ & -7.080 & -2.694 & $115.6 \%$ \\
\hline 19 & $1 \mathrm{~h}$ & 1 & 100 & 1.00 & $60 \%$ & 0.539 & 0.542 & $14.8 \%$ & -1.544 & -1.083 & $66.8 \%$ \\
\hline 20 & $1 \mathrm{~h}$ & 1 & 100 & 1.00 & $95 \%$ & 0.565 & 0.442 & $21.7 \%$ & -2.307 & -1.433 & $78.4 \%$ \\
\hline 21 & $1 \mathrm{~h}$ & 1 & 200 & 0.25 & $60 \%$ & $-5 E+03$ & $-1 E+02$ & $4.6 \mathrm{E}+01$ & $-6 \mathrm{E}+02$ & $-2 \mathrm{E}+01$ & $8.5 \mathrm{E}+00$ \\
\hline 22 & $1 \mathrm{~h}$ & 1 & 200 & 0.25 & $95 \%$ & $-1 \mathrm{E}+04$ & $-1 \mathrm{E}+02$ & $4.9 \mathrm{E}+01$ & $-7 \mathrm{E}+02$ & $-2 \mathrm{E}+01$ & $9.4 \mathrm{E}+00$ \\
\hline 23 & $1 \mathrm{~h}$ & 1 & 200 & 1.00 & $60 \%$ & 0.538 & 0.548 & $16.1 \%$ & -2.240 & -1.396 & $77.8 \%$ \\
\hline 24 & $1 \mathrm{~h}$ & 1 & 200 & 1.00 & $95 \%$ & 0.551 & 0.594 & $12.7 \%$ & -2.089 & -1.318 & $74.2 \%$ \\
\hline 25 & $2 w$ & 1 & 100 & 0.25 & $60 \%$ & 0.272 & 0.414 & $15.6 \%$ & -0.859 & -0.577 & $46.7 \%$ \\
\hline 26 & $2 w$ & 1 & 100 & 0.25 & $95 \%$ & 0.307 & 0.422 & $14.7 \%$ & -0.779 & -0.494 & $43.5 \%$ \\
\hline 27 & $2 w$ & 1 & 100 & 1.00 & $60 \%$ & 0.230 & 0.300 & $18.2 \%$ & -0.501 & -0.368 & $39.8 \%$ \\
\hline 28 & $2 w$ & 1 & 100 & 1.00 & $95 \%$ & 0.220 & 0.268 & $19.4 \%$ & -0.475 & -0.359 & $39.6 \%$ \\
\hline 29 & $2 \mathrm{w}$ & 1 & 200 & 0.25 & $60 \%$ & 0.296 & 0.460 & $14.6 \%$ & -0.757 & -0.527 & $45.2 \%$ \\
\hline 30 & $2 w$ & 1 & 200 & 0.25 & $95 \%$ & 0.377 & 0.485 & $13.8 \%$ & -0.916 & -0.564 & $45.7 \%$ \\
\hline 31 & $2 w$ & 1 & 200 & 1.00 & $60 \%$ & 0.207 & 0.285 & $18.8 \%$ & -0.493 & -0.348 & $39.0 \%$ \\
\hline 32 & $2 w$ & 1 & 200 & 1.00 & $95 \%$ & 0.202 & 0.278 & $18.9 \%$ & -0.525 & -0.382 & $40.3 \%$ \\
\hline
\end{tabular}


Table 6. Parameter assignments and error metrics for all the configurations of Experiment 3 (Indiantown Run), Scenario 1 (July $\left.2^{\text {th }}, 2009\right)$. Configuration 0 corresponds to the default model. All other errors are computed using the OPTIMISTS ensemble mean.

\begin{tabular}{|c|c|c|c|c|c|c|c|c|c|}
\hline \multirow{2}{*}{ Config. } & \multirow{2}{*}{$\Delta t$} & \multirow{2}{*}{$p_{\text {samp }}$} & \multirow{2}{*}{$g$} & \multicolumn{3}{|c|}{ Assimilation period } & \multicolumn{3}{|c|}{ Forecast period } \\
\hline & & & & $\mathrm{NSE}_{\ell 2}$ & $\mathrm{NSE}_{\ell 1}$ & MARE & $\mathrm{NSE}_{\ell 2}$ & $\mathrm{NSE}_{\ell 1}$ & MARE \\
\hline 0 & -- No dat & assimila & on -- & 0.478 & -0.015 & $24.9 \%$ & 0.022 & -0.003 & $54.3 \%$ \\
\hline 1 & $1 \mathrm{~h}$ & 0.4 & 0.5 & $-1 \mathrm{E}+05$ & $-2 \mathrm{E}+02$ & $5.2 \mathrm{E}+01$ & 0.359 & 0.286 & $60.6 \%$ \\
\hline 2 & $1 \mathrm{~h}$ & 0.4 & 1.0 & $-5 \mathrm{E}+03$ & $-3 \mathrm{E}+01$ & $8.3 \mathrm{E}+00$ & 0.366 & 0.271 & $63.1 \%$ \\
\hline 3 & $1 \mathrm{~h}$ & 1.0 & 0.5 & -3.682 & -1.729 & $64.1 \%$ & 0.358 & -0.069 & $107.8 \%$ \\
\hline 4 & $1 \mathrm{~h}$ & 1.0 & 1.0 & -2.038 & -1.146 & $50.9 \%$ & 0.371 & 0.031 & $94.3 \%$ \\
\hline 5 & $6 \mathrm{~h}$ & 0.4 & 0.5 & $-6 E+02$ & $-1 \mathrm{E}+01$ & $2.9 \mathrm{E}+00$ & 0.354 & 0.307 & $57.1 \%$ \\
\hline 6 & $6 \mathrm{~h}$ & 0.4 & 1.0 & $-9 \mathrm{E}+02$ & $-1 \mathrm{E}+01$ & $317.4 \%$ & 0.357 & 0.291 & $59.9 \%$ \\
\hline 7 & $6 \mathrm{~h}$ & 1.0 & 0.5 & -2.107 & -1.230 & $52.0 \%$ & 0.357 & 0.086 & $84.0 \%$ \\
\hline 8 & $6 \mathrm{~h}$ & 1.0 & 1.0 & -1.431 & -0.889 & $43.0 \%$ & 0.361 & 0.292 & $60.1 \%$ \\
\hline 9 & $1 \mathrm{~d}$ & 0.4 & 0.5 & $-2 \mathrm{E}+02$ & -4.030 & $119.4 \%$ & 0.351 & 0.312 & $56.1 \%$ \\
\hline 10 & $1 \mathrm{~d}$ & 0.4 & 1.0 & $-2 \mathrm{E}+03$ & -6.205 & $171.3 \%$ & 0.350 & 0.340 & $52.3 \%$ \\
\hline 11 & $1 \mathrm{~d}$ & 1.0 & 0.5 & -1.406 & -0.891 & $42.8 \%$ & 0.353 & 0.325 & $55.0 \%$ \\
\hline 12 & $1 \mathrm{~d}$ & 1.0 & 1.0 & -1.440 & -0.893 & $42.9 \%$ & 0.352 & 0.315 & $56.6 \%$ \\
\hline 13 & $3.5 \mathrm{~d}$ & 0.4 & 0.5 & $-3 E+03$ & -7.748 & $2.1 \mathrm{E}+00$ & 0.337 & 0.311 & $54.2 \%$ \\
\hline 14 & $3.5 \mathrm{~d}$ & 0.4 & 1.0 & $-6 E+01$ & -1.096 & $49.0 \%$ & 0.328 & 0.337 & $50.2 \%$ \\
\hline 15 & $3.5 \mathrm{~d}$ & 1.0 & 0.5 & 0.224 & 0.067 & $22.1 \%$ & 0.331 & 0.306 & $55.4 \%$ \\
\hline 16 & $3.5 \mathrm{~d}$ & 1.0 & 1.0 & 0.058 & -0.095 & $25.6 \%$ & 0.333 & 0.360 & $48.8 \%$ \\
\hline 17 & $2 w$ & 0.4 & 0.5 & $-8 \mathrm{E}+01$ & -1.527 & $59.6 \%$ & 0.339 & 0.326 & $54.1 \%$ \\
\hline 18 & $2 \mathrm{w}$ & 0.4 & 1.0 & 0.133 & -0.190 & $28.1 \%$ & 0.333 & 0.357 & $49.0 \%$ \\
\hline 19 & $2 w$ & 1.0 & 0.5 & 0.399 & 0.242 & $16.3 \%$ & 0.301 & 0.394 & $40.2 \%$ \\
\hline 20 & $2 w$ & 1.0 & 1.0 & 0.560 & 0.340 & $14.4 \%$ & 0.308 & 0.389 & $41.3 \%$ \\
\hline 21 & $4 w$ & 0.4 & 0.5 & -0.743 & -0.735 & $42.0 \%$ & 0.355 & 0.112 & $79.6 \%$ \\
\hline 22 & $4 \mathrm{w}$ & 0.4 & 1.0 & -1.469 & -1.161 & $52.2 \%$ & 0.358 & 0.047 & $87.4 \%$ \\
\hline 23 & $4 \mathrm{w}$ & 1.0 & 0.5 & -2.873 & -1.763 & $66.4 \%$ & 0.355 & 0.033 & $90.8 \%$ \\
\hline 24 & $4 w$ & 1.0 & 1.0 & -0.577 & -0.622 & $39.2 \%$ & 0.351 & 0.159 & $74.2 \%$ \\
\hline
\end{tabular}


Table 7. Parameter assignments and error metrics for all the configurations of Experiment 3 (Indiantown Run), Scenario 2 (August $\left.2^{\text {th }}, 2009\right)$. Configuration 0 corresponds to the default model. All other errors are computed using the OPTIMISTS ensemble mean.

\begin{tabular}{|c|c|c|c|c|c|c|c|c|c|}
\hline \multirow{2}{*}{ Config. } & \multirow{2}{*}{$\Delta t$} & \multirow{2}{*}{$p_{\text {samp }}$} & \multirow{2}{*}{$g$} & \multicolumn{3}{|c|}{ Assimilation period } & \multicolumn{3}{|c|}{ Forecast period } \\
\hline & & & & $\mathrm{NSE}_{\ell 2}$ & $\mathrm{NSE}_{\ell 1}$ & MARE & $\mathrm{NSE}_{\ell 2}$ & $\mathrm{NSE}_{\ell 1}$ & MARE \\
\hline 0 & -- No dat & assimila & n -- & -0.926 & -0.963 & $68.0 \%$ & -0.428 & -0.733 & $58.3 \%$ \\
\hline 1 & $1 \mathrm{~h}$ & 0.4 & 0.5 & $-1 \mathrm{E}+03$ & $-1 \mathrm{E}+01$ & $6.2 \mathrm{E}+00$ & -0.133 & -0.204 & $37.1 \%$ \\
\hline 2 & $1 \mathrm{~h}$ & 0.4 & 1.0 & $-6 \mathrm{E}+02$ & -8.948 & $4.4 \mathrm{E}+00$ & -1.516 & -0.967 & $60.3 \%$ \\
\hline 3 & $1 \mathrm{~h}$ & 1.0 & 0.5 & 0.604 & 0.686 & $8.0 \%$ & -0.352 & -0.459 & $46.3 \%$ \\
\hline 4 & $1 \mathrm{~h}$ & 1.0 & 1.0 & 0.664 & 0.596 & $14.0 \%$ & -1.591 & -1.133 & $68.3 \%$ \\
\hline 5 & $6 \mathrm{~h}$ & 0.4 & 0.5 & $-1 \mathrm{E}+02$ & -4.849 & $2.7 \mathrm{E}+00$ & -1.270 & -0.769 & $52.7 \%$ \\
\hline 6 & $6 \mathrm{~h}$ & 0.4 & 1.0 & $-2 \mathrm{E}+01$ & -0.391 & $57.4 \%$ & -0.371 & -0.393 & $42.1 \%$ \\
\hline 7 & $6 \mathrm{~h}$ & 1.0 & 0.5 & 0.529 & 0.634 & $8.6 \%$ & -1.098 & -0.679 & $49.6 \%$ \\
\hline 8 & $6 \mathrm{~h}$ & 1.0 & 1.0 & 0.494 & 0.606 & $9.2 \%$ & -0.388 & -0.460 & $45.5 \%$ \\
\hline 9 & $1 \mathrm{~d}$ & 0.4 & 0.5 & -7.399 & 0.020 & $39.8 \%$ & -0.830 & -0.563 & $46.2 \%$ \\
\hline 10 & $1 \mathrm{~d}$ & 0.4 & 1.0 & 0.475 & 0.444 & $14.3 \%$ & -0.213 & -0.286 & $38.6 \%$ \\
\hline 11 & $1 \mathrm{~d}$ & 1.0 & 0.5 & 0.398 & 0.432 & $14.7 \%$ & -1.096 & -0.717 & $51.5 \%$ \\
\hline 12 & $1 \mathrm{~d}$ & 1.0 & 1.0 & 0.453 & 0.512 & $11.9 \%$ & -0.656 & -0.501 & $44.9 \%$ \\
\hline 13 & $3.5 \mathrm{~d}$ & 0.4 & 0.5 & -1.056 & 0.047 & $27.5 \%$ & -0.706 & -0.465 & $42.7 \%$ \\
\hline 14 & $3.5 \mathrm{~d}$ & 0.4 & 1.0 & 0.296 & 0.366 & $15.6 \%$ & -0.690 & -0.437 & $41.6 \%$ \\
\hline 15 & $3.5 \mathrm{~d}$ & 1.0 & 0.5 & 0.028 & 0.076 & $24.9 \%$ & -0.454 & -0.402 & $41.9 \%$ \\
\hline 16 & $3.5 \mathrm{~d}$ & 1.0 & 1.0 & 0.122 & 0.149 & $22.7 \%$ & -0.522 & -0.376 & $40.0 \%$ \\
\hline 17 & $2 \mathrm{w}$ & 0.4 & 0.5 & $-2 \mathrm{E}+01$ & -0.396 & $50.0 \%$ & 0.094 & -0.044 & $30.5 \%$ \\
\hline 18 & $2 w$ & 0.4 & 1.0 & -0.002 & -0.062 & $31.7 \%$ & 0.358 & 0.140 & $25.3 \%$ \\
\hline 19 & $2 \mathrm{w}$ & 1.0 & 0.5 & -0.157 & -0.171 & $35.0 \%$ & 0.340 & 0.108 & $26.4 \%$ \\
\hline 20 & $2 w$ & 1.0 & 1.0 & 0.032 & -0.024 & $30.2 \%$ & 0.301 & 0.109 & $26.0 \%$ \\
\hline 21 & $4 \mathrm{w}$ & 0.4 & 0.5 & 0.379 & 0.491 & $11.4 \%$ & -1.398 & -0.877 & $56.6 \%$ \\
\hline 22 & $4 w$ & 0.4 & 1.0 & 0.330 & 0.424 & $13.4 \%$ & -1.281 & -0.834 & $55.4 \%$ \\
\hline 23 & $4 \mathrm{w}$ & 1.0 & 0.5 & 0.308 & 0.397 & $14.1 \%$ & -0.907 & -0.653 & $49.8 \%$ \\
\hline 24 & $4 w$ & 1.0 & 1.0 & 0.327 & 0.425 & $13.2 \%$ & -1.159 & -0.771 & $53.4 \%$ \\
\hline
\end{tabular}


Table 8. ANOVA table for Experiment 1 for the improvements in NSE $\ell_{2}$ with respect to the default model. DF: degrees of freedom; Adj SS: adjusted sum of squares; Adj MS: adjusted mean sum of squares; Sims.: $n$; Samples: $p_{\text {samp; }}$ F-kernels: $k_{F-c l a s s ~}$.

\begin{tabular}{|c|c|c|c|c|c|}
\hline Source & DF & Adj SS & Adj MS & F-Value & P-Value \\
\hline Model & 33 & 69.586 & 2.109 & 28.910 & 0.000 \\
\hline Scenario & 2 & 66.861 & 33.431 & 458.420 & 0.000 \\
\hline Linear & 5 & 0.914 & 0.183 & 2.510 & 0.039 \\
\hline Objs. & 1 & 0.070 & 0.070 & 0.960 & 0.330 \\
\hline Sims. & 1 & 0.002 & 0.002 & 0.030 & 0.865 \\
\hline TimeStep & 1 & 0.240 & 0.240 & 3.300 & 0.074 \\
\hline Samples & 1 & 0.002 & 0.002 & 0.030 & 0.872 \\
\hline F-kernels & 1 & 0.599 & 0.599 & 8.220 & 0.006 \\
\hline 2-Way Interactions & 10 & 0.622 & 0.062 & 0.850 & 0.581 \\
\hline Objs.*Sims. & 1 & 0.096 & 0.096 & 1.320 & 0.255 \\
\hline Objs. *TimeStep & 1 & 0.041 & 0.041 & 0.560 & 0.457 \\
\hline Objs.*Samples & 1 & 0.021 & 0.021 & 0.290 & 0.595 \\
\hline Objs.*F-kernels & 1 & 0.118 & 0.118 & 1.610 & 0.209 \\
\hline Sims.*TimeStep & 1 & 0.049 & 0.049 & 0.670 & 0.416 \\
\hline Sims.*Samples & 1 & 0.153 & 0.153 & 2.090 & 0.153 \\
\hline Sims.*F-kernels & 1 & 0.087 & 0.087 & 1.190 & 0.279 \\
\hline TimeStep*Samples & 1 & 0.005 & 0.005 & 0.060 & 0.805 \\
\hline TimeStep*F-kernels & 1 & 0.032 & 0.032 & 0.430 & 0.513 \\
\hline Samples*F-kernels & 1 & 0.022 & 0.022 & 0.300 & 0.587 \\
\hline 3-Way Interactions & 10 & 0.848 & 0.085 & 1.160 & 0.332 \\
\hline Objs.*Sims. ${ }^{*}$ TimeStep & 1 & 0.002 & 0.002 & 0.030 & 0.860 \\
\hline Objs.*Sims.*Samples & 1 & 0.027 & 0.027 & 0.370 & 0.543 \\
\hline Objs. ${ }^{*}$ Sims. ${ }^{*}$ F-kernels & 1 & 0.002 & 0.002 & 0.030 & 0.870 \\
\hline Objs.*TimeStep*Samples & 1 & 0.008 & 0.008 & 0.120 & 0.735 \\
\hline Objs. *TimeStep*F-kernels & 1 & 0.095 & 0.095 & 1.300 & 0.259 \\
\hline Objs.*Samples*F-kernels & 1 & 0.022 & 0.022 & 0.300 & 0.588 \\
\hline Sims.*TimeStep*Samples & 1 & 0.299 & 0.299 & 4.090 & 0.047 \\
\hline Sims.*TimeStep*F-kernels & 1 & 0.051 & 0.051 & 0.700 & 0.406 \\
\hline Sims.*Samples*F-kernels & 1 & 0.329 & 0.329 & 4.510 & 0.038 \\
\hline TimeStep*Samples*F-kernels & 1 & 0.014 & 0.014 & 0.190 & 0.668 \\
\hline 4-Way Interactions & 5 & 0.340 & 0.068 & 0.930 & 0.467 \\
\hline Objs.*Sims.*TimeStep*Samples & 1 & 0.010 & 0.010 & 0.140 & 0.714 \\
\hline Objs. *Sims.*TimeStep*F-kernels & 1 & 0.001 & 0.001 & 0.010 & 0.924 \\
\hline Objs.*Sims. *Samples*F-kernels & 1 & 0.027 & 0.027 & 0.360 & 0.548 \\
\hline Objs.*TimeStep*Samples*F-kernels & 1 & 0.086 & 0.086 & 1.180 & 0.282 \\
\hline Sims.*TimeStep*Samples*F-kernels & 1 & 0.217 & 0.217 & 2.970 & 0.090 \\
\hline 5-Way Interaction & 1 & 0.001 & 0.001 & 0.020 & 0.903 \\
\hline Objs. *Sims. $*$ TimeStep*Samples*F-kernels & 1 & 0.001 & 0.001 & 0.020 & 0.903 \\
\hline Error & 62 & 4.521 & 0.073 & & \\
\hline Total & 95 & 74.107 & & & \\
\hline
\end{tabular}


Table 9. ANOVA table for Experiment 1 for the improvements in NSE $_{1}$ with respect to the default model. DF: degrees of freedom; Adj SS: adjusted sum of squares; Adj MS: adjusted mean sum of squares; Sims.: $n$; Samples: $p_{\text {samp; }}$ F-kernels: $k_{F-c l a s s ~}$.

\begin{tabular}{|c|c|c|c|c|c|}
\hline Source & DF & Adj SS & Adj MS & F-Value & P-Value \\
\hline Model & 33 & 20.943 & 0.635 & 47.830 & 0.000 \\
\hline Scenario & 2 & 20.338 & 10.169 & 766.350 & 0.000 \\
\hline Linear & 5 & 0.288 & 0.058 & 4.340 & 0.002 \\
\hline Objs. & 1 & 0.004 & 0.004 & 0.320 & 0.576 \\
\hline Sims. & 1 & 0.002 & 0.002 & 0.130 & 0.720 \\
\hline TimeStep & 1 & 0.034 & 0.034 & 2.530 & 0.117 \\
\hline Samples & 1 & 0.014 & 0.014 & 1.090 & 0.301 \\
\hline F-kernels & 1 & 0.234 & 0.234 & 17.630 & 0.000 \\
\hline 2-Way Interactions & 10 & 0.101 & 0.010 & 0.760 & 0.666 \\
\hline Objs.*Sims. & 1 & 0.008 & 0.008 & 0.590 & 0.446 \\
\hline Objs.*TimeStep & 1 & 0.022 & 0.022 & 1.670 & 0.200 \\
\hline Objs.*Samples & 1 & 0.003 & 0.003 & 0.250 & 0.620 \\
\hline Objs. *F-kernels & 1 & 0.005 & 0.005 & 0.350 & 0.557 \\
\hline Sims.*TimeStep & 1 & 0.004 & 0.004 & 0.270 & 0.607 \\
\hline Sims.*Samples & 1 & 0.009 & 0.009 & 0.690 & 0.411 \\
\hline Sims. ${ }^{* F-k e r n e l s ~}$ & 1 & 0.027 & 0.027 & 2.020 & 0.160 \\
\hline TimeStep*Samples & 1 & 0.014 & 0.014 & 1.030 & 0.315 \\
\hline TimeStep*F-kernels & 1 & 0.010 & 0.010 & 0.740 & 0.393 \\
\hline Samples*F-kernels & 1 & 0.000 & 0.000 & 0.000 & 0.984 \\
\hline 3-Way Interactions & 10 & 0.151 & 0.015 & 1.140 & 0.348 \\
\hline Objs.*Sims. ${ }^{*}$ TimeStep & 1 & 0.003 & 0.003 & 0.200 & 0.657 \\
\hline Objs. *Sims.*Samples & 1 & 0.004 & 0.004 & 0.300 & 0.585 \\
\hline Objs. ${ }^{*}$ Sims. ${ }^{*}$ F-kernels & 1 & 0.000 & 0.000 & 0.020 & 0.898 \\
\hline Objs.*TimeStep*Samples & 1 & 0.002 & 0.002 & 0.120 & 0.728 \\
\hline Objs.*TimeStep*F-kernels & 1 & 0.040 & 0.040 & 3.030 & 0.086 \\
\hline Objs.*Samples*F-kernels & 1 & 0.004 & 0.004 & 0.270 & 0.604 \\
\hline Sims.*TimeStep*Samples & 1 & 0.021 & 0.021 & 1.600 & 0.211 \\
\hline Sims.*TimeStep*F-kernels & 1 & 0.025 & 0.025 & 1.850 & 0.178 \\
\hline Sims. *Samples*F-kernels & 1 & 0.051 & 0.051 & 3.840 & 0.055 \\
\hline TimeStep*Samples*F-kernels & 1 & 0.002 & 0.002 & 0.170 & 0.683 \\
\hline 4-Way Interactions & 5 & 0.065 & 0.013 & 0.980 & 0.434 \\
\hline Objs.*Sims.*TimeStep*Samples & 1 & 0.007 & 0.007 & 0.500 & 0.480 \\
\hline Objs. *Sims.*TimeStep*F-kernels & 1 & 0.000 & 0.000 & 0.010 & 0.931 \\
\hline Objs. *Sims.*Samples*F-kernels & 1 & 0.000 & 0.000 & 0.010 & 0.922 \\
\hline Objs. *TimeStep*Samples*F-kernels & 1 & 0.005 & 0.005 & 0.400 & 0.532 \\
\hline Sims.*TimeStep*Samples*F-kernels & 1 & 0.053 & 0.053 & 4.010 & 0.050 \\
\hline 5-Way Interaction & 1 & 0.001 & 0.001 & 0.040 & 0.845 \\
\hline Objs. *Sims. *TimeStep*Samples*F-kernels & 1 & 0.001 & 0.001 & 0.040 & 0.845 \\
\hline Error & 62 & 0.823 & 0.013 & & \\
\hline Total & 95 & 21.766 & & & \\
\hline
\end{tabular}


Table 10. ANOVA table for Experiment 1 for the improvements in MARE with respect to the default model. DF: degrees of freedom; Adj SS: adjusted sum of squares; Adj MS: adjusted mean sum of squares; Sims.: $n$; Samples: $p_{\text {samp; }}$ F-kernels: $k_{F-c l a s s ~}$.

\begin{tabular}{|c|c|c|c|c|c|}
\hline Source & DF & Adj SS & Adj MS & F-Value & P-Value \\
\hline Model & 33 & 5.556 & 0.168 & 9.240 & 0.000 \\
\hline Scenario & 2 & 4.935 & 2.468 & 135.470 & 0.000 \\
\hline Linear & 5 & 0.383 & 0.077 & 4.210 & 0.002 \\
\hline Objs. & 1 & 0.009 & 0.009 & 0.500 & 0.484 \\
\hline Sims. & 1 & 0.008 & 0.008 & 0.470 & 0.497 \\
\hline TimeStep & 1 & 0.003 & 0.003 & 0.140 & 0.708 \\
\hline Samples & 1 & 0.006 & 0.006 & 0.320 & 0.575 \\
\hline F-kernels & 1 & 0.357 & 0.357 & 19.630 & 0.000 \\
\hline 2-Way Interactions & 10 & 0.136 & 0.014 & 0.740 & 0.680 \\
\hline Objs.*Sims. & 1 & 0.000 & 0.000 & 0.010 & 0.922 \\
\hline Objs.*TimeStep & 1 & 0.030 & 0.030 & 1.630 & 0.207 \\
\hline Objs.*Samples & 1 & 0.000 & 0.000 & 0.020 & 0.902 \\
\hline Objs. *F-kernels & 1 & 0.022 & 0.022 & 1.230 & 0.272 \\
\hline Sims.*TimeStep & 1 & 0.007 & 0.007 & 0.410 & 0.527 \\
\hline Sims.*Samples & 1 & 0.003 & 0.003 & 0.140 & 0.708 \\
\hline Sims. *F-kernels & 1 & 0.009 & 0.009 & 0.480 & 0.489 \\
\hline TimeStep*Samples & 1 & 0.010 & 0.010 & 0.540 & 0.464 \\
\hline TimeStep*F-kernels & 1 & 0.039 & 0.039 & 2.150 & 0.147 \\
\hline Samples*F-kernels & 1 & 0.015 & 0.015 & 0.840 & 0.363 \\
\hline 3-Way Interactions & 10 & 0.085 & 0.008 & 0.470 & 0.906 \\
\hline Objs.*Sims.*TimeStep & 1 & 0.000 & 0.000 & 0.000 & 0.981 \\
\hline Objs.*Sims.*Samples & 1 & 0.001 & 0.001 & 0.050 & 0.824 \\
\hline Objs. ${ }^{*}$ Sims. ${ }^{*}$ F-kernels & 1 & 0.004 & 0.004 & 0.210 & 0.647 \\
\hline Objs.*TimeStep*Samples & 1 & 0.004 & 0.004 & 0.240 & 0.627 \\
\hline Objs.*TimeStep*F-kernels & 1 & 0.028 & 0.028 & 1.540 & 0.219 \\
\hline Objs.*Samples*F-kernels & 1 & 0.001 & 0.001 & 0.070 & 0.785 \\
\hline Sims.*TimeStep*Samples & 1 & 0.017 & 0.017 & 0.930 & 0.339 \\
\hline Sims.*TimeStep*F-kernels & 1 & 0.004 & 0.004 & 0.250 & 0.622 \\
\hline Sims. *Samples*F-kernels & 1 & 0.009 & 0.009 & 0.470 & 0.495 \\
\hline TimeStep*Samples*F-kernels & 1 & 0.016 & 0.016 & 0.890 & 0.348 \\
\hline 4-Way Interactions & 5 & 0.015 & 0.003 & 0.160 & 0.975 \\
\hline Objs.*Sims.*TimeStep*Samples & 1 & 0.000 & 0.000 & 0.020 & 0.892 \\
\hline Objs. ${ }^{* S i m s .}{ }^{*}$ TimeStep*F-kernels & 1 & 0.000 & 0.000 & 0.020 & 0.886 \\
\hline Objs. ${ }^{*}$ Sims. ${ }^{*}$ Samples*F-kernels & 1 & 0.001 & 0.001 & 0.040 & 0.839 \\
\hline Objs.*TimeStep*Samples*F-kernels & 1 & 0.000 & 0.000 & 0.010 & 0.936 \\
\hline Sims.*TimeStep*Samples*F-kernels & 1 & 0.013 & 0.013 & 0.730 & 0.397 \\
\hline 5-Way Interaction & 1 & 0.002 & 0.002 & 0.120 & 0.728 \\
\hline Objs. ${ }^{*}$ Sims. ${ }^{*}$ TimeStep*Samples*F-kernels & 1 & 0.002 & 0.002 & 0.120 & 0.728 \\
\hline Error & 62 & 1.129 & 0.018 & & \\
\hline Total & 95 & 6.685 & & & \\
\hline
\end{tabular}


Table 11. ANOVA table for Experiment 2 for the improvements in NSE $\ell_{2}$ with respect to the default model. DF: degrees of freedom; Adj SS: adjusted sum of squares; Adj MS: adjusted mean sum of squares; Candidates: $n$; Roots: $w_{\text {root }}$.

\begin{tabular}{|c|c|c|c|c|c|}
\hline Source & DF & Adj SS & Adj MS & F-Value & P-Value \\
\hline Model & 32 & 65.769 & 2.055 & 3.450 & 0.000 \\
\hline Scenario & 1 & 11.325 & 11.325 & 19.020 & 0.000 \\
\hline Linear & 5 & 40.151 & 8.030 & 13.490 & 0.000 \\
\hline Time_step & 1 & 25.442 & 25.442 & 42.730 & 0.000 \\
\hline Objectives & 1 & 7.834 & 7.834 & 13.160 & 0.001 \\
\hline Candidates & 1 & 0.866 & 0.866 & 1.450 & 0.237 \\
\hline Optimization & 1 & 5.274 & 5.274 & 8.860 & 0.006 \\
\hline Roots & 1 & 0.735 & 0.735 & 1.230 & 0.275 \\
\hline 2-Way Interactions & 10 & 9.786 & 0.979 & 1.640 & 0.140 \\
\hline Time_step*Objectives & 1 & 1.096 & 1.096 & 1.840 & 0.185 \\
\hline Time_step*Candidates & 1 & 0.697 & 0.697 & 1.170 & 0.288 \\
\hline Time_step*Optimization & 1 & 3.586 & 3.586 & 6.020 & 0.020 \\
\hline Time_step*Roots & 1 & 0.575 & 0.575 & 0.970 & 0.333 \\
\hline Objectives $*$ Candidates & 1 & 0.395 & 0.395 & 0.660 & 0.421 \\
\hline Objectives*Optimization & 1 & 1.635 & 1.635 & 2.750 & 0.108 \\
\hline Objectives*Roots & 1 & 0.801 & 0.801 & 1.350 & 0.255 \\
\hline Candidates*Optimization & 1 & 0.522 & 0.522 & 0.880 & 0.356 \\
\hline Candidates*Roots & 1 & 0.015 & 0.015 & 0.020 & 0.876 \\
\hline Optimization*Roots & 1 & 0.464 & 0.464 & 0.780 & 0.384 \\
\hline 3-Way Interactions & 10 & 3.644 & 0.364 & 0.610 & 0.792 \\
\hline Time_step*Objectives*Candidates & 1 & 0.475 & 0.475 & 0.800 & 0.379 \\
\hline Time_step*Objectives*Optimization & 1 & 1.025 & 1.025 & 1.720 & 0.199 \\
\hline Time_step*Objectives*Roots & 1 & 0.581 & 0.581 & 0.980 & 0.331 \\
\hline Time_step*Candidates*Optimization & 1 & 0.372 & 0.372 & 0.620 & 0.435 \\
\hline Time_step*Candidates*Roots & 1 & 0.000 & 0.000 & 0.000 & 0.980 \\
\hline Time_step*Optimization*Roots & 1 & 0.664 & 0.664 & 1.120 & 0.299 \\
\hline Objectives*Candidates*Optimization & 1 & 0.296 & 0.296 & 0.500 & 0.486 \\
\hline Objectives*Candidates*Roots & 1 & 0.025 & 0.025 & 0.040 & 0.839 \\
\hline Objectives*Optimization*Roots & 1 & 0.172 & 0.172 & 0.290 & 0.595 \\
\hline Candidates*Optimization*Roots & 1 & 0.034 & 0.034 & 0.060 & 0.813 \\
\hline 4-Way Interactions & 5 & 0.861 & 0.172 & 0.290 & 0.915 \\
\hline Time_step*Objectives*Candidates*Optimization & 1 & 0.437 & 0.437 & 0.730 & 0.398 \\
\hline Time_step*Objectives*Candidates*Roots & 1 & 0.028 & 0.028 & 0.050 & 0.831 \\
\hline Time_step*Objectives*Optimization*Roots & 1 & 0.267 & 0.267 & 0.450 & 0.508 \\
\hline Time_step*Candidates*Optimization*Roots & 1 & 0.073 & 0.073 & 0.120 & 0.728 \\
\hline Objectives*Candidates*Optimization*Roots & 1 & 0.056 & 0.056 & 0.090 & 0.761 \\
\hline 5-Way Interaction & 1 & 0.003 & 0.003 & 0.010 & 0.944 \\
\hline Time_step*Objectives*Candidates*Optimization*Roots & 1 & 0.003 & 0.003 & 0.010 & 0.944 \\
\hline Error & 31 & 18.457 & 0.595 & & \\
\hline Total & 63 & 84.226 & & & \\
\hline
\end{tabular}


Table 12. ANOVA table for Experiment 2 for the improvements in NSE $\varepsilon_{\ell}$ with respect to the default model. DF: degrees of freedom; Adj SS: adjusted sum of squares; Adj MS: adjusted mean sum of squares; Candidates: $n$; Roots: $w_{\text {root }}$.

\begin{tabular}{|c|c|c|c|c|c|}
\hline Source & DF & Adj SS & Adj MS & F-Value & P-Value \\
\hline Model & 32 & 39.898 & 1.247 & 13.330 & 0.000 \\
\hline Scenario & 1 & 0.105 & 0.105 & 1.120 & 0.298 \\
\hline Linear & 5 & 30.924 & 6.185 & 66.140 & 0.000 \\
\hline Time_step & 1 & 23.271 & 23.271 & 248.860 & 0.000 \\
\hline Objectives & 1 & 3.714 & 3.714 & 39.720 & 0.000 \\
\hline Candidates & 1 & 0.208 & 0.208 & 2.220 & 0.146 \\
\hline Optimization & 1 & 2.923 & 2.923 & 31.260 & 0.000 \\
\hline Roots & 1 & 0.807 & 0.807 & 8.630 & 0.006 \\
\hline 2-Way Interactions & 10 & 6.188 & 0.619 & 6.620 & 0.000 \\
\hline Time_step*Objectives & 1 & 0.836 & 0.836 & 8.930 & 0.005 \\
\hline Time_step*Candidates & 1 & 0.128 & 0.128 & 1.360 & 0.252 \\
\hline Time_step*Optimization & 1 & 2.229 & 2.229 & 23.840 & 0.000 \\
\hline Time_step*Roots & 1 & 0.665 & 0.665 & 7.110 & 0.012 \\
\hline Objectives*Candidates & 1 & 0.022 & 0.022 & 0.230 & 0.635 \\
\hline Objectives*Optimization & 1 & 1.077 & 1.077 & 11.520 & 0.002 \\
\hline Objectives*Roots & 1 & 0.651 & 0.651 & 6.960 & 0.013 \\
\hline Candidates* Optimization & 1 & 0.079 & 0.079 & 0.850 & 0.364 \\
\hline Candidates*Roots & 1 & 0.002 & 0.002 & 0.020 & 0.900 \\
\hline Optimization*Roots & 1 & 0.500 & 0.500 & 5.350 & 0.028 \\
\hline 3-Way Interactions & 10 & 2.244 & 0.224 & 2.400 & 0.030 \\
\hline Time_step*Objectives*Candidates & 1 & 0.050 & 0.050 & 0.530 & 0.470 \\
\hline Time_step*Objectives*Optimization & 1 & 0.825 & 0.825 & 8.820 & 0.006 \\
\hline Time_step*Objectives*Roots & 1 & 0.530 & 0.530 & 5.660 & 0.024 \\
\hline Time_step*Candidates*Optimization & 1 & 0.046 & 0.046 & 0.500 & 0.487 \\
\hline Time_step*Candidates*Roots & 1 & 0.001 & 0.001 & 0.010 & 0.942 \\
\hline Time_step*Optimization*Roots & 1 & 0.566 & 0.566 & 6.050 & 0.020 \\
\hline Objectives*Candidates*Optimization & 1 & 0.014 & 0.014 & 0.150 & 0.703 \\
\hline Objectives*Candidates*Roots & 1 & 0.000 & 0.000 & 0.000 & 0.963 \\
\hline Objectives*Optimization*Roots & 1 & 0.211 & 0.211 & 2.250 & 0.143 \\
\hline Candidates*Optimization*Roots & 1 & 0.003 & 0.003 & 0.030 & 0.867 \\
\hline 4-Way Interactions & 5 & 0.412 & 0.082 & 0.880 & 0.506 \\
\hline Time_step*Objectives*Candidates*Optimization & 1 & 0.030 & 0.030 & 0.320 & 0.576 \\
\hline Time_step*Objectives*Candidates*Roots & 1 & 0.000 & 0.000 & 0.000 & 0.992 \\
\hline Time_step*Objectives*Optimization*Roots & 1 & 0.290 & 0.290 & 3.100 & 0.088 \\
\hline Time_step*Candidates*Optimization*Roots & 1 & 0.016 & 0.016 & 0.170 & 0.685 \\
\hline Objectives*Candidates*Optimization*Roots & 1 & 0.076 & 0.076 & 0.810 & 0.374 \\
\hline 5-Way Interaction & 1 & 0.026 & 0.026 & 0.280 & 0.603 \\
\hline Time_step*Objectives*Candidates*Optimization*Roots & 1 & 0.026 & 0.026 & 0.280 & 0.603 \\
\hline Error & 31 & 2.899 & 0.094 & & \\
\hline Total & 63 & 42.797 & & & \\
\hline
\end{tabular}


Table 13. ANOVA table for Experiment 2 for the improvements in MARE with respect to the default model. DF: degrees of freedom; Adj SS: adjusted sum of squares; Adj MS: adjusted mean sum of squares; Candidates: $n$; Roots: $w_{\text {root }}$.

\begin{tabular}{|c|c|c|c|c|c|}
\hline Source & DF & Adj SS & Adj MS & F-Value & P-Value \\
\hline Model & 32 & 27.446 & 0.858 & 16.570 & 0.000 \\
\hline Scenario & 1 & 2.191 & 2.191 & 42.320 & 0.000 \\
\hline Linear & 5 & 18.024 & 3.605 & 69.630 & 0.000 \\
\hline Time_step & 1 & 13.747 & 13.747 & 265.540 & 0.000 \\
\hline Objectives & 1 & 1.943 & 1.943 & 37.530 & 0.000 \\
\hline Candidates & 1 & 0.254 & 0.254 & 4.910 & 0.034 \\
\hline Optimization & 1 & 1.567 & 1.567 & 30.270 & 0.000 \\
\hline Roots & 1 & 0.513 & 0.513 & 9.910 & 0.004 \\
\hline 2-Way Interactions & 10 & 5.046 & 0.505 & 9.750 & 0.000 \\
\hline Time_step*Objectives & 1 & 1.148 & 1.148 & 22.180 & 0.000 \\
\hline Time_step*Candidates & 1 & 0.186 & 0.186 & 3.600 & 0.067 \\
\hline Time_step*Optimization & 1 & 1.448 & 1.448 & 27.970 & 0.000 \\
\hline Time_step*Roots & 1 & 0.502 & 0.502 & 9.700 & 0.004 \\
\hline Objectives*Candidates & 1 & 0.108 & 0.108 & 2.090 & 0.159 \\
\hline Objectives*Optimization & 1 & 1.001 & 1.001 & 19.320 & 0.000 \\
\hline Objectives*Roots & 1 & 0.223 & 0.223 & 4.300 & 0.047 \\
\hline Candidates* Optimization & 1 & 0.118 & 0.118 & 2.270 & 0.142 \\
\hline Candidates*Roots & 1 & 0.005 & 0.005 & 0.100 & 0.756 \\
\hline Optimization*Roots & 1 & 0.308 & 0.308 & 5.950 & 0.021 \\
\hline 3-Way Interactions & 10 & 1.880 & 0.188 & 3.630 & 0.003 \\
\hline Time_step*Objectives $*$ Candidates & 1 & 0.145 & 0.145 & 2.800 & 0.105 \\
\hline Time_step*Objectives*Optimization & 1 & 0.910 & 0.910 & 17.580 & 0.000 \\
\hline Time_step*Objectives*Roots & 1 & 0.201 & 0.201 & 3.880 & 0.058 \\
\hline Time_step*Candidates*Optimization & 1 & 0.117 & 0.117 & 2.260 & 0.143 \\
\hline Time_step*Candidates*Roots & 1 & 0.008 & 0.008 & 0.150 & 0.703 \\
\hline Time_step*Optimization*Roots & 1 & 0.294 & 0.294 & 5.690 & 0.023 \\
\hline Objectives*Candidates* Optimization & 1 & 0.112 & 0.112 & 2.170 & 0.151 \\
\hline Objectives $*$ Candidates $*$ Roots & 1 & 0.016 & 0.016 & 0.310 & 0.580 \\
\hline Objectives*Optimization*Roots & 1 & 0.064 & 0.064 & 1.240 & 0.274 \\
\hline Candidates*Optimization*Roots & 1 & 0.012 & 0.012 & 0.230 & 0.638 \\
\hline 4-Way Interactions & 5 & 0.253 & 0.051 & 0.980 & 0.448 \\
\hline Time_step*Objectives*Candidates*Optimization & 1 & 0.100 & 0.100 & 1.930 & 0.175 \\
\hline Time_step*Objectives*Candidates*Roots & 1 & 0.009 & 0.009 & 0.170 & 0.686 \\
\hline Time_step*Objectives*Optimization*Roots & 1 & 0.063 & 0.063 & 1.220 & 0.278 \\
\hline Time_step*Candidates*Optimization*Roots & 1 & 0.016 & 0.016 & 0.300 & 0.586 \\
\hline Objectives*Candidates*Optimization*Roots & 1 & 0.066 & 0.066 & 1.270 & 0.269 \\
\hline 5-Way Interaction & 1 & 0.053 & 0.053 & 1.020 & 0.321 \\
\hline Time_step*Objectives*Candidates*Optimization*Roots & 1 & 0.053 & 0.053 & 1.020 & 0.321 \\
\hline Error & 31 & 1.605 & 0.052 & & \\
\hline Total & 63 & 29.051 & & & \\
\hline
\end{tabular}


Table 14. ANOVA table for Experiment 3 for the improvements in NSE $\varepsilon_{2}$ with respect to the default model. DF: degrees of freedom; Adj SS: adjusted sum of squares; Adj MS: adjusted mean sum of squares; Samples: $p_{\text {samp }}$.

\begin{tabular}{lrrrrr}
\hline Source & DF & Adj SS & Adj MS & F-Value & P-Value \\
\hline Model & 24 & 7.368 & 0.307 & 1.710 & 0.102 \\
Scenario & 1 & 3.551 & 3.551 & 19.770 & 0.000 \\
Linear & 7 & 2.313 & 0.330 & 1.840 & 0.128 \\
$\quad$ Time_step & 5 & 2.310 & 0.462 & 2.570 & 0.055 \\
Samples & 1 & 0.002 & 0.002 & 0.010 & 0.915 \\
Greed & 1 & 0.000 & 0.000 & 0.000 & 0.973 \\
2-Way Interactions & 11 & 1.483 & 0.135 & 0.750 & 0.682 \\
$\quad$ Time_step*Samples & 5 & 0.144 & 0.029 & 0.160 & 0.975 \\
Time_step*Greed & 5 & 1.321 & 0.264 & 1.470 & 0.238 \\
Samples*Greed & 1 & 0.019 & 0.019 & 0.110 & 0.748 \\
3-Way Interactions & 5 & 0.021 & 0.004 & 0.020 & 1.000 \\
$\quad$ Time_step*Samples*Greed & 5 & 0.021 & 0.004 & 0.020 & 1.000 \\
Error & 23 & 4.132 & 0.180 & & \\
Total & 47 & 11.500 & & & \\
\hline
\end{tabular}

5 Table 15. ANOVA table for Experiment 3 for the improvements in NSE $E_{\ell 1}$ with respect to the default model. DF: degrees of freedom; Adj SS: adjusted sum of squares; Adj MS: adjusted mean sum of squares; Samples: $p_{\text {samp }}$.

\begin{tabular}{lrrrrr}
\hline Source & DF & Adj SS & Adj MS & F-Value & P-Value \\
\hline Model & 24 & 2.158 & 0.090 & 2.290 & 0.026 \\
Scenario & 1 & 0.000 & 0.000 & 0.000 & 0.971 \\
Linear & 7 & 1.644 & 0.235 & 5.980 & 0.000 \\
$\quad$ Time_step & 5 & 1.625 & 0.325 & 8.270 & 0.000 \\
$\quad$ Samples & 1 & 0.017 & 0.017 & 0.440 & 0.514 \\
Greed & 1 & 0.002 & 0.002 & 0.040 & 0.843 \\
2-Way Interactions & 11 & 0.501 & 0.046 & 1.160 & 0.366 \\
$\quad$ Time_step*Samples & 5 & 0.156 & 0.031 & 0.790 & 0.564 \\
$\quad$ Time_step*Greed & 5 & 0.344 & 0.069 & 1.750 & 0.163 \\
$\quad$ Samples*Greed & 1 & 0.000 & 0.000 & 0.000 & 0.999 \\
3-Way Interactions & 5 & 0.013 & 0.003 & 0.070 & 0.997 \\
$\quad$ Time_step*Samples*Greed & 5 & 0.013 & 0.003 & 0.070 & 0.997 \\
Error & 23 & 0.904 & 0.039 & & \\
Total & 47 & 3.062 & & & \\
\hline
\end{tabular}


Table 16. ANOVA table for Experiment 3 for the improvements in MARE with respect to the default model. DF: degrees of freedom; Adj SS: adjusted sum of squares; Adj MS: adjusted mean sum of squares; Samples: $p_{\text {samp}}$.

\begin{tabular}{lrrrrr}
\hline Source & DF & Adj SS & Adj MS & F-Value & P-Value \\
\hline Model & 24 & 1.438 & 0.060 & 6.780 & 0.000 \\
$\quad$ Scenario & 1 & 0.643 & 0.643 & 72.820 & 0.000 \\
Linear & 7 & 0.630 & 0.090 & 10.190 & 0.000 \\
$\quad$ Time_step & 5 & 0.605 & 0.121 & 13.700 & 0.000 \\
Samples & 1 & 0.021 & 0.021 & 2.320 & 0.141 \\
$\quad$ Greed & 1 & 0.005 & 0.005 & 0.530 & 0.473 \\
2-Way Interactions & 11 & 0.152 & 0.014 & 1.570 & 0.175 \\
$\quad$ Time_step*Samples & 5 & 0.115 & 0.023 & 2.610 & 0.052 \\
$\quad$ Time_step*Greed & 5 & 0.033 & 0.007 & 0.760 & 0.590 \\
$\quad$ Samples*Greed & 1 & 0.004 & 0.004 & 0.430 & 0.519 \\
3-Way Interactions & 5 & 0.012 & 0.002 & 0.270 & 0.925 \\
$\quad$ Time_step*Samples*Greed & 5 & 0.012 & 0.002 & 0.270 & 0.925 \\
Error & 23 & 0.203 & 0.009 & & \\
Total & 47 & 1.642 & & & \\
\hline
\end{tabular}

